\title{
Autocatalytic backbone N- methylation in a family of ribosomal peptide natural products
}

\section{Journal Article}

\section{Author(s):}

van der Velden, Niels S.; Kälin, Noemi; Helf, Maximilian J.; Piel, Jörn; Freeman, Michael F.; Künzler, Markus (iD

Publication date:

2017-08

Permanent link:

https://doi.org/10.3929/ethz-b-000168811

Rights / license:

Creative Commons Attribution 4.0 International

Originally published in:

Nature Chemical Biology 13, https://doi.org/10.1038/nchembio.2393

\section{Funding acknowledgement:}

146992 - Biosynthetic studies on proteusins, a peptide family with unprecedented modifications (SNF) 613981 - Synthetic Biology for the production of functional peptides (EC) 
1 Autocatalytic backbone $\mathrm{N}$-methylation in a family of ribosomal peptide

2 natural products

3

4

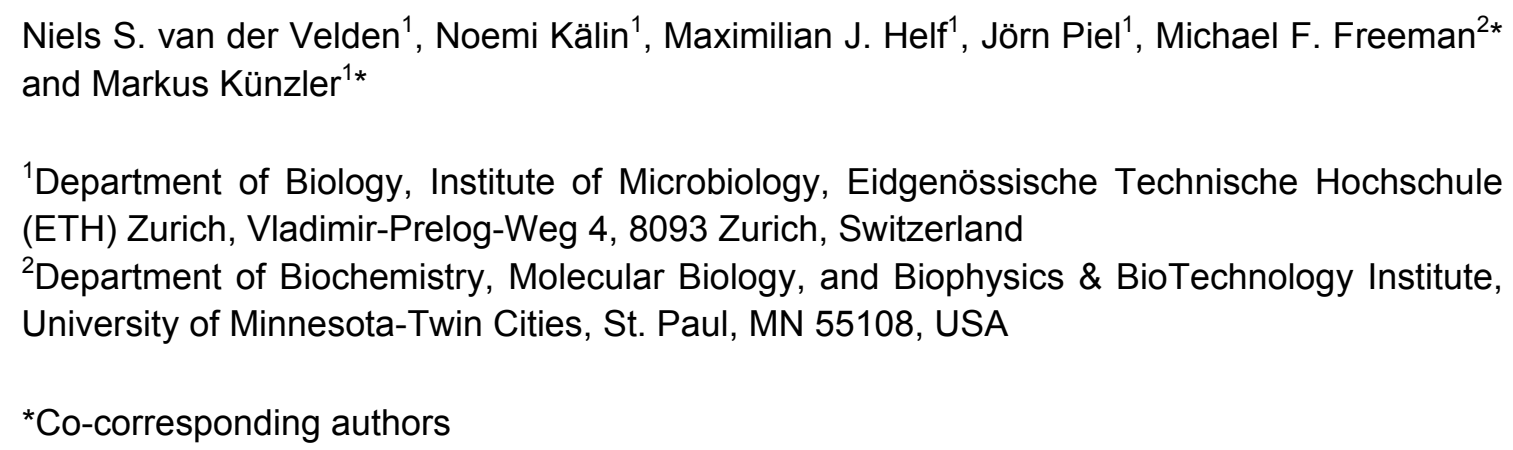

Peptide backbone $\mathrm{N}$-methylations as in cyclosporin $\mathrm{A}$ have been an exclusive characteristic of non-ribosomal peptides. We have identified the first posttranslationally modified peptide or protein harboring internal $\alpha-\mathrm{N}$-methylations through discovery of the genetic locus for the omphalotins, cyclic $\mathrm{N}$-methylated peptides produced by the fungus Omphalotus olearius. We show iterative autocatalytic activity of an $\mathrm{N}$-methyltransferase fused to its peptide substrate is the signature of a new family of ribosomally encoded metabolites.

The fidelity and complexity of protein- and peptide-mediated interactions is predominantly achieved through the vast chemical space provided by the 20 canonical proteinogenic amino acids. This space is extended by posttranslational modifications (PTMs) such as phosphorylation, glycosylation, acetylation, amidation, and many others ${ }^{1}$. An expanded repertoire of PTMs can be found in ribosomally synthesized and posttranslationally modified peptides (RiPPs), a class of natural products that is found in all kingdoms of life and invokes an extensive array of biological activities ${ }^{2}$. RiPPs are produced by translation of protein/peptide precursors followed by posttranslational modification of these precursors by additional enzymes. Many of these accessory proteins are presumably recruited by the $\mathrm{N}$ terminal leader sequence of the precursor to amend the C-terminal peptide core ${ }^{2}$. Proteolytic release and export of the core affords the active metabolite.

In contrast to RiPP biosynthesis, nonribosomal peptide synthetases (NRPSs) create structurally diverse peptides through direct incorporation of non-proteinogenic amino acids ${ }^{3}$. This feat is achieved in an assembly line-like fashion where individual modules within giant multimodular NRPS proteins are responsible for the activation, incorporation, and optional further modification of amino acids.

Recent insights into RiPP enzymes have begun to challenge the view that certain residues and peptide modifications are only accessible to NRPS pathways ${ }^{4}$. Nonetheless, while the methylation of peptide $\mathrm{N}$-termini and side chains have been observed in RiPPs ${ }^{2}$, backbone $\mathrm{N}$-methylation has only been found in NRPS-derived peptides and shown to occur prior to amide bond formation ${ }^{5}$. These modifications have been demonstrated to improve therapeutic peptide metabolic stability, membrane permeability, target selectivity, affinity and oral bioavailability ${ }^{5}$. Despite these favorable properties, backbone $\alpha-\mathrm{N}-$ methylation has to our knowledge not been reported as a PTM in any naturally occurring ribosomal peptide or protein $^{5}$. 
Cyclic peptides such as the nematotoxic omphalotins from the basidiomycete $O$. olearius, in which 9 of the 12 backbone nitrogens are methylated, were therefore assumed to be products of an $\operatorname{NRPS}^{6}$ (Fig. 1a). However, a basic local alignment search tool (BLAST) search of the circularly permuted omphalotin peptide sequence (WVIVVGVIGVIG) against the $O$. olearius genome revealed a protein-encoding gene (ophA) containing a stretch of codons consistent with the omphalotin peptide scaffold (core peptide) as part of a gene cluster (Fig. 1b). Surprisingly, the OphA sequence $\mathrm{N}$-terminal of the core peptide (leader sequence) comprises 399 amino acid residues and contains a domain with sequence homology to SAM-dependent methyltransferases (Fig. 1c). Such a protein domain architecture has, to our knowledge, never been observed before in RiPP precursors. Recognition motifs present in other RiPP family leader sequences, namely the RiPP precursor peptide recognition element (RRE) or the a-helical sequence conserved in microviridins ${ }^{7}$, were not detected in OphA.

To test whether OphA was both the genetic source of omphalotins and the methyltransferase responsible for $\alpha-\mathrm{N}$-methylation, ophA cDNA was cloned and the gene encoding OphA with an $\mathrm{N}$-terminal histidine affinity tag was expressed in $E$. coli and purified via metal chelate chromatography (Supplementary Results, Supplementary Fig. 1). After one day of expression, electrospray ionization time of flight mass spectrometry (ESI-TOF-MS) analysis of recombinant full-length OphA showed a mass shift corresponding to ten methylations (Supplementary Note). In contrast, omission of the C-terminal omphalotin-encoding region of OphA (OphA-NP) resulted in the expected mass of the unmodified recombinant protein (Supplementary Note). Detailed peptide fingerprinting of trypsin-digested OphA was performed using high performance liquid chromatography coupled tandem mass spectrometry with high-energy collision dissociation (HPLC-MS/MS HCD). OphA expressions from one to five days revealed a suite of differentially methylated species with up to 11 methylations sequentially extending from the $\mathrm{N}$ - to $\mathrm{C}$-terminus of the precursor core peptide (Fig. 2a, Supplementary Note). The pattern of methylation was the same as in the natural products, with two additional methylations in the sequence C-terminal of the core peptide (Cterminal recognition sequence). Electron-transfer dissociation (ETD) fragmentation unambiguously localized the methylation sites to the backbone nitrogens (Fig. 2b).

Based on homology of OphA with homodimeric SAM-dependent methyltransferases, we hypothesized that residues Y98 and S129 might be located within the OphA SAM-binding pocket (Supplementary Fig. 2). In agreement with this hypothesis, alanine substitutions for Y98 and S129 indeed abolished methylation (Supplementary Fig. 3a-c). These results suggest that the methyltransferase domain of OphA is required for the observed methylation of the omphalotin peptide sequence and that this activity is likely SAM-dependent. Gel filtration experiments suggested OphA associates into homodimers (Supplementary Fig. 1), incurring the possibility that the apparent automethylation occurred either intra- or intermolecularly. In order to distinguish between these two possibilities, the inactive mutants OphA S129A and OphA Y98A were coexpressed in trans-complementation experiments with the catalytically active core peptide mutant OphA V5T. After 36-hour expression in E. coli, the otherwise inactive OphA mutants harbored up to ten $\alpha-\mathrm{N}$-methylations, revealing the capability of OphA to act in trans (Supplementary Fig. 3d-f). Attempts to further address this issue, through observing and characterizing in vitro activity of purified OphA, have thus far been unsuccessful despite observing activity in cell-free extracts with or without supplementation of S-adenosylmethionine (Supplementary Fig. 4); this has been seen in 
other RiPP systems ${ }^{8}$. Future experiments will be necessary to further validate that OphA catalyses intermolecular $\alpha-\mathrm{N}$-methylation and to test whether the enzyme accepts peptide substrates unbound to the leader sequence.

To determine OphA sequence requirements for methylation, we introduced several mutations into the flanking regions of the OphA core peptide. Removal of the C-terminal recognition sequence (OphA-NF) resulted in incomplete methylation near the C-terminus (Supplementary Note). Sequences following RiPP core peptides have been postulated to be able to replace leader sequences in the case of bottromycins ${ }^{9}$, and have been shown to be involved in other PTMs such as dehydration/decarboxylation ${ }^{10}$ and macrocyclization ${ }^{11-13}$. On the other hand, mutation of residues 392 through 396 to incorporate a TEV cleavage site (OphA-TEV) preceding the core peptide resulted in a methylation-competent precursor (Supplementary Note). Thus, there appears to be a moderate sequence flexibility for the flanking residues of the core peptide sequence, opening the possibility of engineering cleavable $\mathrm{N}$-methylated linear peptides without the necessity of additional biosynthetic enzymes.

We exchanged the omphalotin core peptide in OphA with sequences closely resembling two naturally occurring, backbone $\mathrm{N}$-methylated therapeutic peptides: the NRPS-derived cyclosporin $A$ (CycA), used as an immunosuppressant drug in organ transplantations ${ }^{14}$, and dictyonamide A (DicA), shown to be a cyclin-dependent kinase 4 inhibitor ${ }^{15}$. HPLC-MS/MS HCD analysis of the trypsinized fragments comprising the CycA- and DicA-like cores revealed up to five methylations for the CycA-like core (Fig. 2c) and up to nine for the DicAlike peptide fragment (Fig. 2d) upon expression for five days in E. coli. Whole-protein ESITOF-MS of these samples revealed the most abundant species were the two- and five-times methylated CycA-like peptide and the three- and eight-times methylated DicA-like peptides (Supplementary Note). Of note, the core methylation patterns of these hybrids did not fully correspond to the patterns of the natural products (Fig. 2). Nonetheless, these data are exciting proof-of-principle findings since synthetic production of custom backbone $\mathrm{N}$ methylated peptides in large quantities is still troublesome due to low coupling yields and side reactions ${ }^{16}$. While these types of peptides can be produced by in vitro translation using custom-loaded aminoacyl-tRNAs ${ }^{17}$, the yields from this process are limited. Thus, a scalable in vivo method for the production of backbone methylated peptides, such as with this system capable of producing differentially methylated species on alternative OphA core sequences, would be an attractive strategy.

All of the above results strongly suggest that omphalotins are not NRPS products but the founding member of a novel family of RiPPs. Analysis of the ophA locus in the O. olearius genome revealed that the gene is part of a gene cluster with enzymes possibly involved in tailoring and maturation of the core peptide (Fig. 1b). Two putative P450 monooxygenases and an O-acyltransferase are likely involved in posttranslational modification of the precursor to create the peptide scaffolds found in omphalotins B-1 ${ }^{18}$ (Supplementary Fig. 5). Macrocyclization of omphalotins is likely mediated by a putative prolyl oligopeptidase; endopeptidases have been shown to cyclise peptides in bacteria, fungi, and plants as seen in the biosynthesis of patellamides ${ }^{11}$, amatoxins ${ }^{12}$ and cyclotides ${ }^{13}$. Experiments to confirm the function of the other enzymes flanking ophA in the O. olearius genome are ongoing. 
BLAST searches of other sequenced fungal genomes using the proteins encoded in the omphalotin gene cluster as queries revealed a homologous gene cluster in the basidiomycete Dendrothele bispora CBS 962.96 (Supplementary Fig. 6a). As an initial proof of concept, the putative precursor gene found in D. bispora (dbophA) was synthesized (Supplementary Fig. 6b). DbOphA was functionally competent in that it methylated its core peptide, whose sequence is highly homologous to the omphalotins, in a similar pattern to OphA (Supplementary Note).

Examples of autocatalysis are widespread in nature and range from autoproteolysis, autophosphorylation, and automethylation to isopeptide bond formation and pseudocofactor/chromophore maturation ${ }^{19}$. Intermolecular autocatalysis has been observed less frequently but has been described in the maturation of proteases like thrombin ${ }^{20}$ and capsid assembly of bacteriophage $\mathrm{HK} 97^{21}$. However, nearly all of these processes have one of two functions: either they modulate the catalytic activity or stabilize the structure of the respective enzyme. By contrast, OphA is an enzyme that contains the structural information of a metabolite and the catalytic domain to modify its structure.

In summary, we demonstrate that the omphalotins are the founding members of a fourth family of fungal RiPPs besides the amatoxins ${ }^{22}$, dikaritins (comprising ustiloxins and phomopsins $)^{23,24}$ and epichloëcyclins ${ }^{25}$. The hallmark of this newly described family is $\mathrm{N}$ methylation of the peptide backbone accomplished by a methyltransferase domain encoded within the leader of the peptide precursor protein. The methyltransferase domain in OphA appears to act iteratively, in an N-to-C-terminal consecutive manner, on the core peptide sequence that is located at the C-terminus of the precursor protein and destined to be cleaved off for the generation of the mature natural product. Although we are unaware of examples where a RiPP modifying enzyme was directly fused to a core peptide to circumvent the need for a leader, several research groups have successfully engineered artificial fusions of RiPP modifying enzymes to leader sequences in order to observe in trans modification of core peptides ${ }^{26,27}$. To our knowledge, such a self-modifying reaction as part of a natural product biosynthetic pathway is without precedent.

For this newly discovered family of RiPPs, we propose the name 'Borosins' after the ancient mythological symbol Ouroboros, a serpent biting into its own tail. Based on our findings that the identified methyltransferase is functional in the $E$. coli cytoplasm and appears to be promiscuous with regard to acceptor peptide sequence specificity, this RiPP family opens the possibility to produce backbone $\mathrm{N}$-methylated peptide libraries in $E$. coli for the identification of new bioactive peptides.

\section{Methods}

Methods, including statements of data availability and any associated accession codes and references, are available in the online version of the paper.

\section{Acknowledgements}

We thank Markus Aebi for helpful discussions and continuous support, Jeremy Thorner for helpful suggestions regarding the manuscript, the functional genomics center Zürich for ESITOF-MS measurements, Alexander Brachmann for help with HPLC-MS/MS HCD measurements, Aman Imani for performing cell-free experiments and Chia-wei Lin for help with HPLC-MS/MS ETD measurements. We are grateful to László Nagy and Francis Martin 
for sharing unpublished genome and transcriptome data of Dendrothele bispora CBS 962.96 and Marasmius fiardii PR-910, respectively, produced and made publicly available by the US Department of Energy Joint Genome Institute (DOE JGI). This work was supported by ETH Zürich (M.K., J.P.), the University of Minnesota (M.F.F.), the Swiss National Science Foundation (31003A_146992) (J.P.), and the EU 7th framework program (SYNPEPTIDE) (J.P.).

\section{Author contributions}

M.K. initiated and supervised the project. M.F.F. and N.S.V. designed the experiments. N.S.V. and N.K. conducted the experiments. N.S.V., M.F.F. and M.J.H. analysed the data. All authors discussed the results. M.F.F., N.S.V. and M.K. wrote the manuscript. J.P. edited the manuscript and provided experimental expertise.

\section{Competing financial interests}

M.K., M.F.F., N.S.V., N.K. and J.P., are inventors on European Patent Application no. EP16164245.9 filed by ETH Zürich titled "Novel multiply Backbone N-methyl transferase and use thereof".

\section{Additional information}

Any supplementary information, chemical compound information and source data are available in the online version of the paper. Reprints and permissions information is available online at http://www.nature.com/reprints/index.html. Correspondence and requests for materials should be addressed to M.K. and M.F.F.

\section{References}

1. Walsh, C. T., Garneau-Tsodikova, S. \& Gatto, G. J. Protein posttranslational modifications: the chemistry of proteome diversifications. Angew. Chem. Int. Ed. Engl. 44, 7342-72 (2005).

2. Arnison, P. G. et al. Ribosomally synthesized and post-translationally modified peptide natural products: overview and recommendations for a universal nomenclature. Nat. Prod. Rep. 30, 108-160 (2013).

3. Walsh, C. T., O'Brien, R. V \& Khosla, C. Nonproteinogenic amino acid building blocks for nonribosomal peptide and hybrid polyketide scaffolds. Angew. Chem. Int. Ed. Engl. 52, 7098-124 (2013).

4. Freeman, M. F. et al. Metagenome mining reveals polytheonamides as posttranslationally modified ribosomal peptides. Science 338, 387-90 (2012).

5. Chatterjee, J., Rechenmacher, F. \& Kessler, H. N-methylation of peptides and proteins: an important element for modulating biological functions. Angew. Chem. Int. Ed. Engl. 52, 254-269 (2013).

6. Sterner, O., Etzel, W., Mayer, A. \& Anke, H. Omphalotin, a new cyclic peptide with potent nematicidal activity from Omphalotus olearius II. Isolation and structure determination. Nat. Prod. Lett. 10, 33-38 (1997).

7. Li, K., Condurso, H. L., Li, G., Ding, Y. \& Bruner, S. D. Structural basis for precursor protein-directed ribosomal peptide macrocyclization. Nat. Chem. Biol. 12, 973-979 (2016).

8. Garg, N., Salazar-Ocampo, L. M. A. \& van der Donk, W. A. In vitro activity of the nisin dehydratase NisB. Proc. Natl. Acad. Sci. 110, 7258-63 (2013). 
9. Gomez-Escribano, J. P., Song, L., Bibb, M. J. \& Challis, G. L. Posttranslational $\beta-$ methylation and macrolactamidination in the biosynthesis of the bottromycin complex of ribosomal peptide antibiotics. Chem. Sci. 3, 3522 (2012).

10. Ghodge, S. V., Biernat, K. A., Bassett, S. J., Redinbo, M. R. \& Bowers, A. A. Posttranslational claisen condensation and decarboxylation en route to the bicyclic core of pantocin A. J. Am. Chem. Soc. 138, 5487-5490 (2016).

11. Koehnke, J. et al. The mechanism of patellamide macrocyclization revealed by the characterization of the PatG macrocyclase domain. Nat. Struct. Biol. 19, 767-772 (2012).

12. Luo, H. et al. Peptide macrocyclization catalyzed by a prolyl oligopeptidase involved in a-amanitin biosynthesis. Chem. Biol. 21, 1610-1617 (2014).

13. Craik, D. J., Daly, N. L., Bond, T. \& Waine, C. Plant cyclotides: A unique family of cyclic and knotted proteins that defines the cyclic cystine knot structural motif. J. Mol. Biol. 294, 1327-1336 (1999).

14. Faulds, D., Goa, K. L. \& Benfield, P. Cyclosporin. A review of its pharmacodynamic and pharmacokinetic properties, and therapeutic use in immunoregulatory disorders. Drugs 45, 953-1040 (1993).

15. Komatsu, K., Shigemori, H. \& Kobayashi, J. Dictyonamides A and B, new peptides from marine-derived fungus. J Org Chem 66, 6189-6192 (2001).

16. Pattabiraman, V. R. \& Bode, J. W. Rethinking amide bond synthesis. Nature 480, 471479 (2011).

17. Maini, R., Umemoto, S. \& Suga, H. Ribosome-mediated synthesis of natural productlike peptides via cell-free translation. Curr. Opin. Chem. Biol. 34, 44-52 (2016).

18. Liermann, J. C. et al. Omphalotins E-I, five oxidatively modified nematicidal cyclopeptides from Omphalotus olearius. European J. Org. Chem 8, 1256-1262 (2009).

19. Ravikiran, B. \& Mahalakshmi, R. Unusual post-translational protein modifications: the benefits of sophistication. RSC Adv. 4, 33958 (2014).

20. Petrovan, R. J. et al. Autocatalytic peptide bond cleavages in prothrombin and meizothrombin. Biochemistry 37, 1185-91 (1998).

21. Duda, R. L. Protein chainmail: catenated protein in viral capsids. Cell 94, 55-60 (1998).

22. Hallen, H. E., Luo, H., Scott-Craig, J. S. \& Walton, J. D. Gene family encoding the major toxins of lethal Amanita mushrooms. Proc. Natl. Acad. Sci. 104, 19097-19101 (2007).

23. Ding, W. et al. Biosynthetic investigation of phomopsins reveals a widespread pathway for ribosomal natural products in Ascomycetes. Proc. Natl. Acad. Sci. 113, 3521-3526 (2016).

24. Koiso, Y. et al. Ustiloxin: a phytotoxin and a mycotoxin from false smuth balls on rice panicles. Tetrahedron Lett. 33, 4157-4160 (1992).

25. Johnson, R. D. et al. A novel family of cyclic oligopeptides derived from ribosomal peptide synthesis of an in planta-induced gene, gigA, in Epichloë endophytes of grasses. Fungal Genet. Biol. 85, 14-24 (2015).

26. Oman, T. J., Knerr, P. J., Bindman, N. A., Velásquez, J. E. \& van der Donk, W. A. An engineered lantibiotic synthetase that does not require a leader peptide on its substrate. J. Am. Chem. Soc. 134, 6952-6955 (2012).

27. Koehnke, J. et al. Structural analysis of leader peptide binding enables leader-free cyanobactin processing. Nat. Chem. Biol. 11, 558-563 (2015). 


\section{Figure legends}

290

291

292

293

294

295

296

297

298

299

300

301

302

303

304

305

306

307

308

309

310

311

312

313

314

315

316

317

318

319

320

321

322

323

324

325

326

327

328

329

330

331

332

333

334

335

Figure 1 I Omphalotin A: Natural product and encoding genetic locus. (a) Structure of omphalotin $A$, with the a-nitrogen methyl groups indicated in green. (b) The putative biosynthetic gene cluster for omphalotin A (further details in Supplementary Table 2). (c) The core sequence (italicized residues 1 to 12) of the precursor protein OphA, encoding the omphalotins, is flanked by a leader (residues 1 to 399) and C-terminal recognition sequence (CRS; residues +1 to +6 ). Due to methyltransferase domain homology (PF00590) within the leader, residue numbering is modified as suggested for RiPPs ${ }^{2}$.

Figure 2 I Observed methylation states of wild-type and hybrid omphalotin precursors. Each peptide sequence represents MS/MS spectra for the C-terminal tryptic fragment of OphA purified from various E. coli expressions. Solid green circles indicate MS/MS confirmation of $\mathrm{N}$-methylated residues; unfilled circles represent detected but undefined $\mathrm{N}$ methylation positions. Schematics of relevant natural products are depicted at the top of the panels. ESI-TOF-MS, HPLC-MS/MS and estimated relative amounts of methylated OphA tryptic fragments are in the Supplementary Note. (a) Methylation of OphA. Zero through 10 methylations (with 10 being most abundant) were observed after one-day expressions, while 11 methylations could only be observed after five days. (b) Confirmation of methylations localized to backbone $\alpha$-nitrogens by HPLC-MS/MS ETD. Observed b-, c- and y-ions of the three-times methylated OphA fragment after four hours of expression are denoted on the omphalotin-encoding peptide structure; the observed methylations are in perfect agreement with the natural product. (c,d) Methylation states of hybrid omphalotin precursors OphACycA and OphA-DicA after five-day expressions, where the omphalotin-encoding sequence is exchanged with a peptide resembling cyclosporin $A(\mathbf{c})$ or dictyonamide $A(\mathbf{d})$. ${ }^{*}=\mathrm{D}-$ alanine; R1 = (2S, 3R, 4R, 6E)-3-hydroxy-4-methyl-2-methylamino-6-octenoic acid; R2 = L- $\alpha$ aminobutyric acid; $A b z=2$-aminobenzoic acid.

\section{Online Methods}

cDNA synthesis and cloning of the ophA precursor. O. olearius strain DSM3398 (DSMZ) was used for RNA extraction and cDNA synthesis. O. olearius mycelium was grown on cellophane disks (Celloclair) on $1.5 \%(\mathrm{w} / \mathrm{v})$ agar plates containing yeast extract maltose agar $(0.4 \%(\mathrm{w} / \mathrm{v})$ yeast extract (Oxoid AG), $1 \%(\mathrm{w} / \mathrm{v})$ malt extract (Oxoid AG), $0.4 \%(\mathrm{w} / \mathrm{v})$ glucose) for 5 days at $28{ }^{\circ} \mathrm{C}$ in the dark. To extract RNA, $8.2 \mathrm{mg}$ of lyophilized mycelium was lysed with $8 \mathrm{mg}$ of $0.5 \mathrm{~mm}$ glass beads in three FastPrep (Thermo Savant) steps of $45 \mathrm{~s}$ at $4.5,5.5$ and 6.5, cooling the sample for $5 \mathrm{~min}$ on ice between each step. RNA was extracted with 1 $\mathrm{ml}$ Qiazol (Qiagen) and $0.2 \mathrm{ml}$ chloroform. After centrifugation at $12000 \mathrm{~g}$ at $4{ }^{\circ} \mathrm{C}$ for $15 \mathrm{~min}$, RNA was recovered in the aqueous phase, loaded and washed on-column using the RNeasy Lipid Tissue Mini Kit (Qiagen) and eluted with RNase-free water. cDNA was synthesized from $2 \mu \mathrm{g}$ of extracted RNA using the Transcriptor-first strand cDNA synthesis kit (Roche Applied Science) following the manufacturer's instructions. The putative coding sequence of the omphalotin precursor gene was amplified from cDNA by PCR using Phusion high-fidelity DNA polymerase (Thermofisher) with primers Fw-OMP and Rv-OMP (Supplementary Table 1). The PCR product was subjected to an A-tailing reaction before it was cloned into the pGEM-T-easy vector (Promega) and transformed to E. coli DH5a. For construction of an $E$. coli expression plasmid, a PCR reaction was performed using the pGEM-T-easy-cDNA plasmid as template and pET24-Fw-OMP or pET24-Fw-HisOMP and pET24-Rv-OMP 
(Supplementary Table 1) as primers. The resulting PCR product encoding an N-terminal His tag was cloned into pET24 (Merck Millipore) using the restriction sites Ndel and Notl (Thermo Scientific) and the resulting plasmids were transformed into E. coli BL21(DE3) (Promega). All DNA constructs were verified by DNA-sequencing (Microsynth AG).

Synthesis and cloning of dbophA. dbophA was synthesized based on publicly available RNA sequencing data that can be found on the JGI website (http://genome.jgi.doe.gov/vista embed/?organism=Denbi1) and codon-optimized for expression in E. coli by Genscript. The codon optimized sequence containing an N-terminal His tag and Ndel and HindllI restriction sites was as follows: $5^{\prime}$-CATATGGAACATCATCATCATCATCATCATCATTCTTCTACTCAA ACCAAACCCGGTTCGCTCATCGTCGTCGGTACAGGCATCGAGAGCATCGGTCAAATGA CGCTCCAAGCCTTATCGTACATTGAAGCTGCTTCCAAAGTCTTCTATTGTGTCATCGACC CTGCGACAGAGGCTTTTATCCTCACCAAGAACAAGAATTGTGTCGACTTGTATCAGTACT ACGACAATGGCAAGTCTAGAATGGATACTTACACCCAAATGGCTGAGCTCATGCTCAAG GAAGTCCGCAATGGCCTCGACGTTGTCGGGGTATTCTATGGCCATCCGGGCGTGTTCG TGAACCCTTCTCACAGGGCATTGGCTATCGCCAGAAGCGAGGGATACCAAGCTCGTAT GCTTCCAGGAGTATCTGCAGAGGACTGCCTCTTTGCCGACTTATGCATCGACCCCTCGA ACCCCGGCTGCTTGACCTACGAAGCATCCGATTTCCTCATCAGAGAGAGACCAGTGAAC GTTCACAGTCACCTCATTCTTTTCCAAGTTGGATGCGTCGGTATCGCAGACTTCAATTTC AGCGGATTCGACAATTCGAAATTTACCATTCTGGTTGACCGTCTCGAGCAAGAATACGG CCCAGACCATACCGTCGTGCATTATATCGCAGCTATGATGCCTCACCAAGATCCCGTCA CCGACAAGTTCACGATCGGACAACTCCGTGAACCCGAAATCGCCAAACGAGTAGGTGG TGTTTCGACTTTTTACATCCCTCCAAAGGCCAGAAAGGATATCAACACCGATATCATCCG ACTCTTGGAATTCCTACCTGCTGGTAAAGTTCCCGACAAACACACCCAAATCTACCCAC CTAATCAATGGGAACCCGACGTGCCTACTCTACCTCCCTATGGACAAAACGAACAGGCT GCTATCACTAGATTGGAAGCTCACGCTCCCCCCGAAGAGTATCAGCCTCTGGCCACTTC CAAAGCTATGACTGACGTCATGACCAAACTAGCTTTGGATCCCAAGGCGCTCGCCGAGT ATAAGGCTGATCATCGAGCCTTCGCTCAGTCTGTTCCTGACTTGACGCCTCAAGAGAGA GCAGCTTTGGAGCTAGGAGATTCATGGGCTATTCGGTGCGCCATGAAGAACATGCCGT CCTCTCTCTTGGAAGCCGCTAGCCAGTCCGTCGAAGAGGCATCCATGAACGGTTTCCC ATGGGTCATCGTCACGGGTATCGTTGGGGTCATCGGATCGGTTGTGAGCAGTGCTTGA AAGCTT-3'. The codon-optimized ORF was recloned into pET24 for expression in E. coli BL21(DE3) using the restriction sites Ndel and HindIII.

Cloning of ophA-NF, -NP, -TEV, -CycA and -DicA. In order to remove the sequence encoding the omphalotin core peptide (ophA-NP) a stop codon was introduced after the proline followed by a Bsal restriction site using ligation of a synthetic adapter consisting of oligonucleotides Fw-OMP-Bsal and Rv-OMP-Bsal (Supplementary Table 1). Primers were annealed by combining $10 \mu \mathrm{l}$ of each oligonucleotide $(100 \mu \mathrm{M})$ and mixing it with $80 \mu \mathrm{l}$ of 10 $\mathrm{mM}$ Tris $\mathrm{pH}$ 7.5. The mixture was subjected to the following program: $5 \mathrm{~min}$ at $98{ }^{\circ} \mathrm{C}$ with a cooling rate of $4{ }^{\circ} \mathrm{C} / \mathrm{s}, 30$ seconds at $80{ }^{\circ} \mathrm{C}$ with a cooling rate of $0.01{ }^{\circ} \mathrm{C} / \mathrm{s}$ and 30 seconds at $40{ }^{\circ} \mathrm{C}$ with a cooling rate of $0.02{ }^{\circ} \mathrm{C} / \mathrm{s}$. The annealed primers were phosphorylated and ligated into pET24 containing ophA and digested with restriction endonucleases $\mathrm{Ncol}$ and Notl (Thermo Scientific) at a vector to insert mole ratio of 1:7. The inserted Bsal site was then used for removal of the C-terminal recognition sequence or to insert sequences coding for CycA and DicA using primers 8 to 13 in Supplementary Table 1. Primer annealing was performed as described above and the synthetic DNA fragments were ligated into pET24ophA containing the introduced Bsal site using restriction sites Bsal and Notl. For the introduction of the TEV cleavage site in the ophA gene the following DNA fragment was 

GGATCCCAAGGCACTCGCCGACTACAAAGCTGATCACCGCGCCTTTGCTCAATCTGTCC CCGACTTGACGCCTCAGGAGCGTGCGGCTTTGGAGCTCGGTGATTCGTGGGCTATTCG TTGCGCGATGAAGAATATGCCCTCGTCGCTCTTGGACGCTGCTCGTGAATCCGGCGAA AACTTGTACTTCCAAGGTTTCCCATGGGTCATCGTCGTTGGTGTTATCGGTGTCATCGG ATAAGAATTC-3:. The fragment was ligated into pET24 containing the ophA ORF using the restriction sites BamHI and EcoRI.

Generation of constructs encoding OphA P399A, V5T, Y98A, and S129A. To generate the ophA mutants, site directed mutagenesis was performed with the Phusion Site-directed Mutagenesis Kit from Thermo Scientific according to the instructions of the manufacturer using primers 14 to 21 (Supplementary Table 1) using pET24-ophA as a template. To clone non-tagged ophA V5T into pCDFDUET-1 (Merck Millipore), a PCR reaction was performed using the pET24-ophA V5T plasmid as template and the primers pCDF-Fw-OphA V5T and pCDF-Rv-OphA V5T (Supplementary Table 1). The resulting PCR product was digested with Bsal and HindIII and cloned into pCDFDUET-1 using the restriction sites Ncol and HindIII.

Purification of His-tagged OphA, OphA variants and DbOphA from E. coli BL21(DE3). E. coli BL21(DE3) transformants containing the various ophA and dbophA expression vectors were pre-cultured overnight in LB medium containing $50 \mu \mathrm{g} / \mathrm{ml}$ kanamycin at $37^{\circ} \mathrm{C} .1$ $\mathrm{ml}$ of pre-culture was diluted in $1 \mathrm{~L}$ of TB medium $(2.4 \%(\mathrm{w} / \mathrm{v})$ yeast extract (Oxoid $\mathrm{AG}), 1.2 \%$ (w/v) tryptone (Oxoid $A G$ ), $0.4 \%$ (w/v) glycerol, $0.17 \mathrm{M} \mathrm{KH}_{2} \mathrm{PO}_{4}$ and $0.72 \mathrm{M} \mathrm{K}_{2} \mathrm{HPO}_{4}$ ) containing $50 \mu \mathrm{g} / \mathrm{ml}$ kanamycin. Cultures were grown at $37{ }^{\circ} \mathrm{C}$ to an $\mathrm{OD}_{600}$ between $1.5-2$ after which they were chilled on ice for $20 \mathrm{~min}$. $0.2 \mathrm{mM}$ IPTG was added and further incubation was performed at $16{ }^{\circ} \mathrm{C}$ for the indicated expression times. Bacterial cells were spun down at $8000 \mathrm{~g}$ for $5 \mathrm{~min}$ at $4{ }^{\circ} \mathrm{C}$, pellets were resuspended in ice-cold buffer $(50 \mathrm{mM}$ HEPES pH 8, 0.1\% (v/v) triton X-100, 10\% (v/v) glycerol) containing $20 \mathrm{mM}$ imidazole and lysed using a French press. Cell debris was removed by centrifugation at $16000 \mathrm{~g}$ for $30 \mathrm{~min}$. The supernatant was incubated with Ni-NTA beads (Thermo Scientific) at $4{ }^{\circ} \mathrm{C}$ for $1 \mathrm{~h}$, washed with 10 column volumes of ice-cold buffer and the protein was finally eluted in buffer containing $400 \mathrm{mM}$ imidazole. Purified protein was concentrated on an Amicon Ultra-4 centrifugal filter device (Millipore) with a molecular weight cutoff of $30 \mathrm{kDa}$, desalted using a PD-10 column (Amersham Biosciences) in buffer without imidazole and flash-frozen in liquid nitrogen before being stored at $-20{ }^{\circ} \mathrm{C}$. Protein concentrations were measured using the Pierce BCA protein assay (Thermo scientific) using a standard curve derived from bovine serum albumin.

Size exclusion chromatography. Size exclusion chromatography was performed using a Superdex-200 Increase column (GE healthcare). The column was calibrated using high and low molecular weight gel filtration standards (GE healthcare) in $50 \mathrm{mM} \mathrm{HEPES} \mathrm{pH} \mathrm{8,} \mathrm{0.1 \%}$ $(\mathrm{v} / \mathrm{v})$ triton X-100, 10\% (v/v) glycerol. The column was pre-equilibrated in the same buffer and OphA $(5 \mathrm{mg} / \mathrm{mL})$ was applied to the column in a total volume of $100 \mu \mathrm{l}$. Flow-through fractions were collected and analyzed by $12 \%(\mathrm{w} / \mathrm{v})$ SDS-PAGE.

HPLC-MS/MS HCD. An Amicon Ultra column (30 kDa cutoff) was washed two times with $\mathrm{dd}_{2} \mathrm{O}$. Protein $(100 \mu \mathrm{g})$ diluted with $\mathrm{ddH}_{2} \mathrm{O}$ to a final volume of $400 \mu \mathrm{L}$ was added. The sample was centrifuged (11000 $\mathrm{g}$ for $5 \mathrm{~min}$ ) and washed with $500 \mu \mathrm{l}$ of $\mathrm{dd}_{2} \mathrm{O}$. A buffer 
containing $50 \mathrm{mM} \mathrm{NH}_{4} \mathrm{HCO}_{3} \mathrm{pH} 8$ was added and the sample was concentrated to $4 \mu \mathrm{g} / \mu \mathrm{L}$. 25 $\mu \mathrm{l}$ was transferred to a glass vial, trypsin (Promega) was added in a molar ratio of 1:70 and the sample incubated for $3 \mathrm{~h}$ at $37^{\circ} \mathrm{C} .3 \mu \mathrm{l}$ of each sample was used for mass spectrometry analysis. HPLC-MS/MS data was recorded on a Thermo Scientific $Q$ Exactive mass spectrometer equipped with a Dionex Ultimate 3000 UHPLC system using a Phenomenex Kinetex $2.6 \mu \mathrm{m} \mathrm{C} 18100 \AA(150 \times 4.6 \mathrm{~mm})$ column heated at $50{ }^{\circ} \mathrm{C}$. Elution was performed with a linear gradient using water with $0.1 \%(\mathrm{v} / \mathrm{v})$ formic acid $(\mathrm{FA})$ (solvent $\mathrm{A}$ ) and acetonitrile $(\mathrm{ACN})$ with $0.1 \%(\mathrm{v} / \mathrm{v})$ FA (solvent $\mathrm{B}$ ) at a flow rate of $0.8 \mathrm{~mL} / \mathrm{min}$. The column was equilibrated with $5 \%$ solvent B for 2 min followed by a linear increase of solvent B to $85 \%$ over $15 \mathrm{~min}$ and a final elution step with $98 \%$ solvent B for $5 \mathrm{~min}$. Mass spectra were acquired in positive ion mode with the following settings; spray voltage $3500 \mathrm{~V}$, capillary temperature at $268.75^{\circ} \mathrm{C}$, probe heater at $437.5^{\circ} \mathrm{C}$ and an S-lens level at 50. Full MS was done at a resolution of 35000 (AGC target 1e6, maximum IT $100 \mathrm{~ms}$, range between 500$1800 \mathrm{~m} / \mathrm{z}$ ) and data-dependent MS/MS was performed at a resolution of 17500 (AGC target at 2e5, maximum IT of 300 ms isolation window 1.2) using a stepped NCE of 16, 20 and 24. The inclusion list contained the mass of the trypsinized fragment encoding the omphalotin peptide (or one of the variants) and its different methylation states. Data were processed using ThermoFisher Xcalibur software.

HPLC-MS/MS ETD. Sample preparation was performed as stated above (see: HPLC-MS/MS HCD). $50 \mu \mathrm{l}$ of trypsinized sample was desalted and purified using C18 ZipTips (Merck Millipore). ZipTips were washed 5 times with $10 \mu \mathrm{l}$ of $90 \%$ ACN with $0.1 \%$ (v/v) FA followed by ten times equilibration with $10 \mu \mathrm{l}$ of $0.1 \%(\mathrm{v} / \mathrm{v}) \mathrm{FA}$. After equilibration, peptides were bound to the column by pipetting $10 \mu$ of sample up and down 10 times and washed 10 times with $10 \mu \mathrm{l}$ of $0.1 \%(\mathrm{v} / \mathrm{v})$ FA. Peptides were eluted in $10 \mu \mathrm{l} 70 \% \mathrm{ACN}$ with $0.1 \%(\mathrm{v} / \mathrm{v}) \mathrm{FA}$ by pipetting up and down ten times followed by an additional elution with $90 \%$ ACN with $0.1 \%$ $(\mathrm{v} / \mathrm{v})$ FA. Eluates were pooled and dried using a speedvac and dissolved in $20 \mu \mathrm{l}$ of $2.5 \%$ ACN with $0.1 \%(v / v)$ FA. $3 \mu$ l of each sample was used for MS analysis. HPLC-MS/MS data was recorded on a LTQ-Orbitrap Fusion mass spectrometer (Thermo Fischer Scientific) coupled to an Easy-nLC 1000 UHPLC system (Thermo Fischer Scientific) using a self-made fritted column $(75 \mu \mathrm{m} \times 150 \mathrm{~mm})$ packed with reverse-phase C18 material (ReproSil-Pur 120 $\mathrm{C} 18-\mathrm{AQ}, 1.9 \mu \mathrm{m}$, Dr. Maisch $\mathrm{GmbH}$ ) heated at $50{ }^{\circ} \mathrm{C}$. Elution was performed with a linear gradient using water with $0.1 \%(\mathrm{v} / \mathrm{v})$ FA (solvent $\mathrm{A}$ ) and ACN with $0.1 \%(\mathrm{v} / \mathrm{v})$ FA (solvent B) at a flow rate of $0.3 \mu \mathrm{L} / \mathrm{min}$. The column was equilibrated with $5 \%$ solvent $B$ for 2 min followed by a linear increase of solvent B to $85 \%$ over $15 \mathrm{~min}$ and a final elution step with $98 \%$ solvent B for 5 min. For MS analysis, one scan cycle comprised of a full scan MS survey spectrum, followed by electron-transfer/higher-energy collision dissociation (EThcD) MS/MS scans with a maximum duty cycle of 3 seconds using a target list. Full-scan MS spectra $(600-2000 \mathrm{~m} / \mathrm{z})$ were acquired in the FT-Orbitrap at a resolution of 120,000 at 400 $\mathrm{m} / \mathrm{z}$, while EThcD MS/MS spectra were recorded in the FT-Orbitrap at a resolution of 30,000 at $400 \mathrm{~m} / \mathrm{z}$. ETD was performed with a target value of 2e5 with supplementary activation energy set at 30. For all experiments, dynamic exclusion was used with 1 repeat count, $30 \mathrm{~s}$ repeat duration, and $10 \mathrm{~s}$ exclusion duration. Data were processed using ThermoFisher Xcalibur software.

ESI-TOF-MS. Determination of total mass by ESI-TOF-MS was performed by the Functional Genomics Center Zürich (FGCZ). In order to get rid of detergent, proteins were precipitated with $20 \%$ (w/v) trichloroacetic acid (TCA), reconstituted in hexafluorisopropanol (HFIP) prior to the analysis. ESI MS data was recorded on a Waters Q-TOF SYNAPT G2 HDMS in a 
mass range between 50 and $5000 \mathrm{Da}$; the sampling cone energy was set at $35-50 \mathrm{~V}$. The $\mathrm{m} / \mathrm{z}$ data were then deconvoluted into MS data using the MaxEnt1 software (Waters).

Trans-complementation assays. E. coli BL21(DE3) containing pCDF-ophA V5T and either the pET24-ophA Y98A or S129A expression vector was grown in LB medium containing a final concentration of $50 \mu \mathrm{g} / \mathrm{ml}$ kanamycin or streptomycin, respectively. Protein expression was induced for $36 \mathrm{~h}$ after which His-tagged OphA-Y98A or OphA-S129A was purified via metal chelate chromatography. Methylation was assessed via HPLC-MS/MS HCD as described above.

490

Cell-free assays. An E. coli BL21(DE3) culture was grown overnight at $37^{\circ} \mathrm{C}$. Bacterial cells were spun down at $8000 \mathrm{~g}$ for $5 \mathrm{~min}$ at $4{ }^{\circ} \mathrm{C}$, pellets were resuspended in ice-cold buffer (50 $\mathrm{mM}$ HEPES $\mathrm{pH} 8,0.1 \%(\mathrm{v} / \mathrm{v})$ triton $\mathrm{X}-100,10 \%$ glycerol) and lysed using a French press. Cell debris was removed by centrifugation at $16000 \mathrm{~g}$ for $30 \mathrm{~min}$. Previously purified OphA expressed for four hours in $E$. coli was added to $300 \mu \mathrm{l}$ of cell lysate to a final concentration of $5 \mu \mathrm{M}$. After three days of incubation at room temperature, $25 \mu \mathrm{l}$ of the cell lysate was transferred to a glass vial, trypsin (Promega) was added in a mole ratio of 1:35 and the sample incubated for three hours at $37^{\circ} \mathrm{C} .15 \mu \mathrm{l}$ of the sample was used for mass spectrometry analysis as described previously.

Data Availability. The data that support the findings of this study are available from the 503 


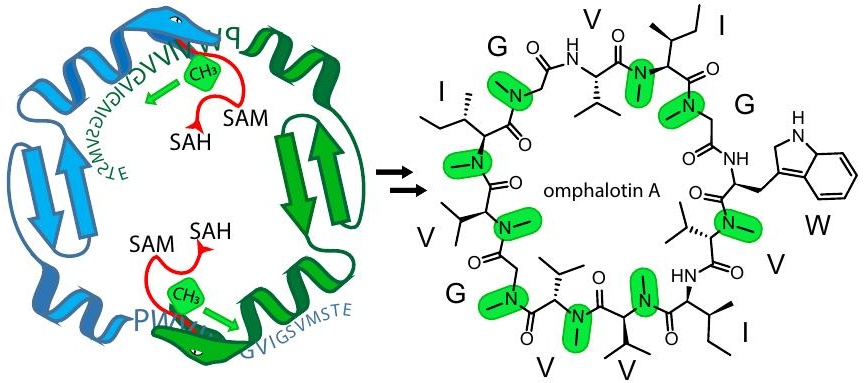




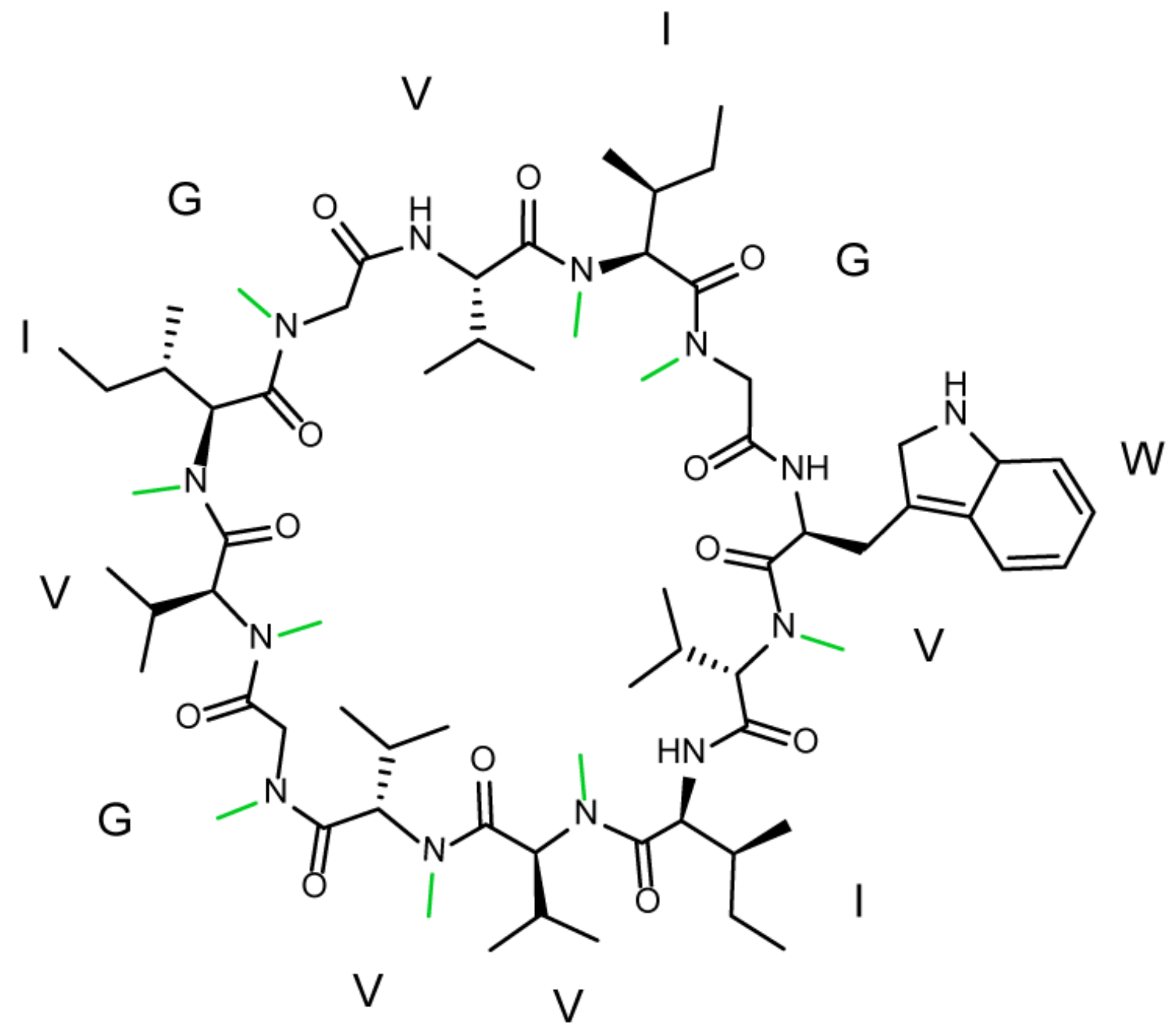

$\begin{array}{lllll}\text { ophB1 ophC ophA ophD ophB2 ophP ophE } & \text { op }\end{array}$

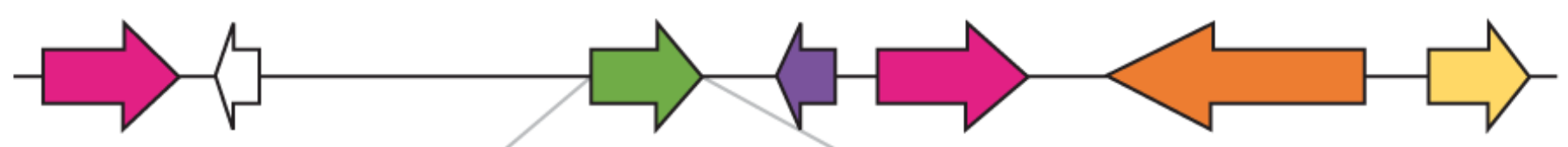

$\square$ Monooxygenase $\square$ NTF2-like $\square$ Methyltransferase $\square$ O-acyltransferase $\quad \overline{1 \mathrm{~kb}}$ $\square$ Prolyloligopeptidase $\square$ F-box/RNI-like

C

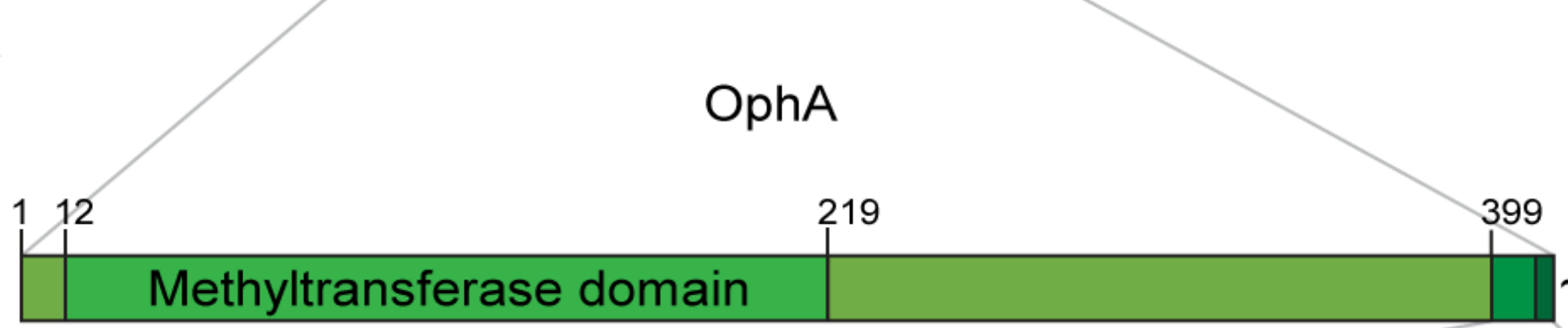




\section{Omphalotin A: $\quad$ LV I VVGVIG VIG-}

ESGEEASQNGF PWV I VVGVIGVIGS VMS TE ESGEE A S QNGF PWVI VVGVIGV IGS DMS TE ESGEEASQNGF PWV I VVGVIGVIGS VMS TE ESGEEASQNGF PWV I VVGVIGV IGS VMSTE ESGEEASQNGF PWV I VVGVIGV I GS VMS TE ESGEEASQNGFPWVI VVGVIGV I GSVMSTE ESGEEASQNGFPWVI VVGVIGVIGSVMSTE ESGEEASQNGFPWVI VVGV I GV I GSVMSTE ESGEEASQNGFPWVI VVGVIGVIGSVMSTE ESGEEASQNGFPWVI VVGVIGVIGSVMSTE ESGEEASQNGFPWVIVVGVIGVIGSVMSTE ESGEEASQNGFPWVIVVGVIGVIGSVMSTE b

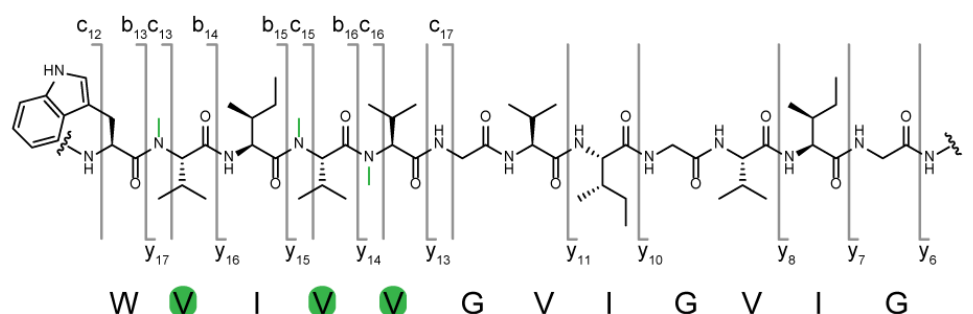

Cyclosporin A:

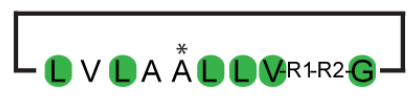

ESGEE A S QNGF PL VLAALL V I VG SVMSTE ESGEEASQNGFPL VLAALL V I VGSVMSTE E SGEEASQNGFPLVLAAL (D)V (1)VGSVMSTE ESGEEASQNGFPL VLAAL LV IVGSVMSTE ESGEEASQNGFPL VLAAL LV I VGSVMSTE

d
A T TVVVVV I VG-Abz
ESGEEASQNGFPATTVVVVVTVG ESGEEASQNGFPATTVVVVVIVG ESGEEASQNGFPAT TVVVVVIVG ESGEEASQNGFPATTVVVVV (1)VG ESGEEASQNGFPATTVVVVVIVG ESGEEASQNGFPATTVVVVVIVG ESGEEASQNGFPATTVVVVVIVG ESGEEASQNGFPATTVVVVVIVG ESGEEASQNGFPATTVVVVVIVG ESGEEASQNGFPATTVVVVVIVG ESGEEASQNGFPATTVVVVVIVG ESGEEASQNGFPATT VVVVVIVG ESGEEASQNGFPATTVVVVVIVG

Position of N-Me confirmed by MS/MS $\bigcirc$ MS/MS detected but undefined N-Me position 